\title{
Developing a new template foreground cleaning method for the LiteBIRD experiment
}

\section{Kiyotomo Ichiki*}

Kobayashi-Maskawa Institute for the Origin of Particles and the Universe, Nagoya University, Chikusa-ku, Nagoya, 464-8602, Japan

E-mail: ichiki@a.phys.nagoya-u.ac.jp

\section{Tetsu Yamashita}

Graduate School of Engineering, Yokohama National University, 79-5 Tokiwadai, Hodogaya-ku, Yokohama 240-8501 JAPAN

E-mail: yamashita-tetsu-df@ynu.jp

\section{Hiroaki Kanai}

Graduate School of Engineering, Yokohama National University, 79-5 Tokiwadai, Hodogaya-ku, Yokohama 240-8501 JAPAN

E-mail: kanai-hiroaki-nw@ynu.jp

\section{Eiichiro Komatsu}

Max Planck Institute for Astrophysics, Karl-Schwarzschild-Str. 1, D-85748 Garching, Germany Kavli Institute for the Physics and Mathematics of the Universe (Kavli IPMU, WPI), Todai Institutes for Advanced Study, The University of Tokyo, Kash iwa 277-8583, Japan

E-mail: komatsu@mpa-garching.mpg.de

\section{Nobuhiko Katayama}

Kavli Institute for the Physics and Mathematics of the Universe (Kavli IPMU, WPI), Todai Institutes for Advanced Study, The University of Tokyo, Kash iwa 277-8583, Japan

E-mail: nobu.katayama@ipmu.jp

Detecting gravitational wave background generated during inflation through B-mode polarization of the cosmic microwave background (CMB) is one of the main scientific goals of future CMB experiments. However, it has already become clear that the synchrotron and thermal dust emissions from our galaxy hinder the cosmological B-mode signal across the sky. Here, assuming only Gaussianity and statistical isotropy and homogeneity of the CMB map, we develop a new template cleaning method taking into account spatial variations of spectral indices of the foreground emissions, and apply it to various foreground models using Monte-Carlo simulation technique. We find that the method successfully remove the unwanted bias in determining the tensor-toscalar ratio $r$ that was found in the simple template cleaning method presented in Katayama and Komatsu 2011. In this poster, we present how small $r$ we will be able to reach according to the frequency band and noise specification proposed for the LiteBird experiment.

The 3rd International Symposium on "Quest for the Origin of Particles and the Universe" 5-7 January 2017

Nagoya University, Japan

\footnotetext{
* Speaker.
} 


\section{Introduction}

Inflation is an accelerating expansion phase in the early universe and offers a solution to the nagging problems in Big-Bang cosmology, namely, the horizon problem, flatness problem, monopole problem and so on. Many models of inflation have been proposed so far, and one observational consequence that most inflation models commonly predict is the existence of gravitational wave background in the universe. Whilst a direct measurement of the gravitational wave background from inflation using laser interferometers seems very difficult with the current technology, an indirect detection through the B-mode polarization of the Cosmic Microwave Background (CMB) may be possible in the near future, provided that the energy scale of inflation is as high as the grand unification scale of particle physics. The amplitude of the inflationary gravitational waves is usually characterized by a parameter $r$, so called the "tensor-to-scalar ratio", which is defined by

$$
r \equiv \frac{2\left\langle\left|h(k)_{+}\right|^{2}+\left|h(k)_{\times}\right|^{2}\right\rangle}{\left\langle|\mathscr{R}(k)|^{2}\right\rangle}
$$

where $h(k)_{+, \times}$and $\mathscr{R}(k)$ denote gravitational-wave amplitudes of two different polarizations and curvature perturbation amplitude, respectively.

From recent observations of the CMB, $r$ is constrained as $r \lesssim 0.12$. At this level, the B-mode polarization signal is dominated by foreground emissions, namely synchrotron and thermal dust emissions, from our galaxy. Therefore, we have to estimate the foregrounds and subtract them to reach the cosmological signal and many methods have been proposed and tested (for a review, see [1]). In Katayama and Komatsu ([2]; hereafter KK11) we used the Planck Sky Model (PSM) to show that the residual foreground due to spatial variation of synchrotron spectral indices yields a bias in the tensor-to-scalar ratio that is as small as $\delta r=6 \times 10^{-3}$. The spatial variation of dust spectral indices was, however, unknown at that time. Now that we have some information about spatial variation of the dust indices from the Planck temperature data, we can revisit the bias in the tensor-to-scalar ratio from dust indices.

In this work we develop new template cleaning methods to mitigate the biases that is introduced due to spatial variation of the spectral indices of foreground emissions. We consider two methods: Method (1) - we assume that both synchrotron and dust emissions show power law spectrum with spatially varying spectral index and Method (2) - we assume that synchrotron emission is modeled as a power-law component while dust emission is described by the modified black body spectrum with spatially varying spectral indices and dust temperature. Below we shall describe our template cleaning methods.

\section{2. methodology}

Let us begin with Method (1), in which we assume that the spectrum of dust emission obeys a power-law. Ignoring synchrotron for the moment for simplicity, we model the Stokes parameters $Q$ and $U$ at a given frequency $v$ as

$$
[Q, U]_{v}(\hat{n})=\operatorname{CMB}(\hat{n})+g_{v}\left(\frac{v}{v_{d}}\right)^{\beta_{\mathrm{d}}(\hat{n})}[Q, U]_{v_{\mathrm{d}}}(\hat{n})+N_{v}(\hat{n}),
$$

where $v_{\mathrm{d}}$ is some reference frequency for dust emission. The Planck data suggest that the spatial variation of $\beta_{\mathrm{d}}(\hat{n})$ is small compared with the mean index $\bar{\beta}_{\mathrm{d}}$. Therefore, we can Taylor expand the spatial dependence of $\beta_{\mathrm{d}}(\hat{n})$ and write the above equation as

$$
[Q, U]_{v}(\hat{n}) \approx \operatorname{CMB}(\hat{n})+g_{v}\left(\frac{v}{v_{d}}\right)^{\bar{\beta}_{\mathrm{d}}}\left(1+\ln \left(\frac{v}{v_{\mathrm{d}}}\right) \delta \beta_{\mathrm{d}}(\hat{n})\right)[Q, U]_{v_{\mathrm{d}}}(\hat{n})+N_{v}(\hat{n}) .
$$


It is the term, $\delta \beta_{\mathrm{d}}(\hat{n})[Q, U]_{V_{\mathrm{d}}}(\hat{n})$, that biases the estimate of the tensor-to-scalar ratio when using the internal template method assuming a constant spectral index $\beta_{\mathrm{d}}$ in KK11. Therefore, we can reduce the bias by estimating $\delta \beta_{\mathrm{d}}(\hat{n})[Q, U]_{V_{\mathrm{d}}}(\hat{n})$ and subtracting it.

To subtract it let us consider the linear combination

$$
\begin{aligned}
& {[Q, U]_{v_{0}}(\hat{n})+\sum_{i=1}^{2} \alpha_{i}[Q, U]_{v_{i}}(\hat{n})=\left(1+\sum_{i=1}^{2} \alpha_{i}\right) \operatorname{CMB}(\hat{n})+N_{v_{0}}(\hat{n})+\sum_{i=1}^{2} \alpha_{i} N_{v_{i}}(\hat{n})} \\
& +\left(d_{1}\left(v_{0}\right)+\sum_{i=1}^{2} d_{1}\left(v_{i}\right) \alpha_{i}\right)[Q, U]_{V_{\mathrm{d}}}(\hat{n})+\left(d_{2}\left(v_{0}\right)+\sum_{i=1}^{2} d_{2}\left(v_{i}\right) \alpha_{i}\right)[Q, U]_{v_{\mathrm{d}}}(\hat{n}) \delta \beta_{\mathrm{d}}(\hat{n}),
\end{aligned}
$$

where

$$
d_{1}(v)=g_{v}\left(\frac{v}{v_{\mathrm{d}}}\right)^{\bar{\beta}_{\mathrm{d}}}, d_{2}(v)=g_{v}\left(\frac{v}{v_{\mathrm{d}}}\right)^{\bar{\beta}_{\mathrm{d}}} \log \left(\frac{v}{v_{\mathrm{d}}}\right)
$$

We can remove the dust emission if the coefficients $\alpha_{i}$ are set to the solution of the equations

$$
d_{j}\left(v_{0}\right)+\sum_{i=1}^{2} d_{j}\left(v_{i}\right) \alpha_{i}=0
$$

for $j=1,2$. In Method (2), we consider a modified black body spectrum for dust emission instead of a power law one. In this case, the above equation is modified to, with $x \equiv h v / k T$,

$$
\begin{aligned}
{[Q, U]_{v}(\hat{n}) } & \approx \operatorname{CMB}(\hat{n})+g_{v}[Q, U]_{\mathrm{d}}(\hat{n})\left(\frac{v}{v_{\mathrm{d}}}\right)^{\overline{\beta_{\mathrm{d}}}} \frac{1}{e^{x}-1}\left[1+\log \left(\frac{v}{v_{\mathrm{d}}}\right) \delta \beta_{\mathrm{d}}(\hat{n})+\frac{x e^{x}}{e^{x}-1} \frac{\delta T(\hat{n})}{\bar{T}}\right] \\
& +N_{v}(\hat{n})
\end{aligned}
$$

and $d_{j}(v)$ are modified to,

$$
d_{1}(v)=g_{v}\left(\frac{v}{v_{\mathrm{d}}}\right)^{\bar{\beta}_{\mathrm{d}}} \frac{1}{e^{x}-1}, d_{2}(v)=d_{1}(v) \log \left(\frac{v}{v_{\mathrm{d}}}\right) .
$$

\section{Foreground maps for the simulation}

In this work we consider two foreground sky models. One is the Planck Sky Model (PSM), in which the synchrotron and thermal dust emissions are modeled as power laws in antenna temperature with spectral indices varying in sky directions. The other is the one component dust model (onecomp) in which thermal dust emission is modeled by the modified black body spectrum with spectral indices and dust temperature varying in sky directions.

\section{Simulation and Result}

We generate hundred mock stokes $\mathrm{Q}$ and $\mathrm{U}$ maps of the CMB at each LiteBIRD band based on the theoretical power spectra $\left(C_{\ell}\right)$, where the B-mode amplitude $C_{\ell}^{\mathrm{BB}}$ is characterized by the free parameter $r$. The foreground maps and instrumental noise are added on them at each band. We then use the method discussed above and maximize the likelihood:

$$
\mathscr{L}\left(r, \bar{\beta}_{\mathrm{d}}, \bar{\beta}_{\mathrm{s}}\right) \propto \frac{\exp \left[-\frac{1}{2}[Q, U]^{T} \boldsymbol{C}^{-1}[Q, U]\right]}{\sqrt{\left|\boldsymbol{C}\left(r, \bar{\beta}_{\mathrm{d}}, \bar{\beta}_{\mathrm{s}}\right)\right|}}
$$

where $[Q, U]$ is a vector of a template-cleaned map and $\boldsymbol{C}$ is a covariance of the same map.

The result is shown in Figure 1, where the estimated tensor-to-scalar ratio $r_{\text {est }}$ is shown against the true (input) one $r_{\text {input }}$. In the left panel of fig. 1, we show results using the method (1) (delta 
map method in the figure). We find that the method successfully removes the foreground and gives unbiased estimations if the foreground emissions are simple power-laws (PSM; blue). However, if the dust emission is modeled by a modified black body spectrum, the method (1) fails to remove the foreground and introduces a significant bias as large as $\delta r \approx 0.1$ (onecomp; red).

We can do better to the onecomp model if we use the method (2), where we model the dust component by a modified black body spectrum accordingly. However, in this case, we find that instrumental noise starts to dominate at the foreground cleaned band because template cleaning method 'cleans' the part of the CMB as well as the foreground, leading to an unwanted noise boost (right panel of Fig. 1, blue). To check this is caused by the instrumental noise, we perform noiseless simulations for which we show the result in magenta in the same panel.
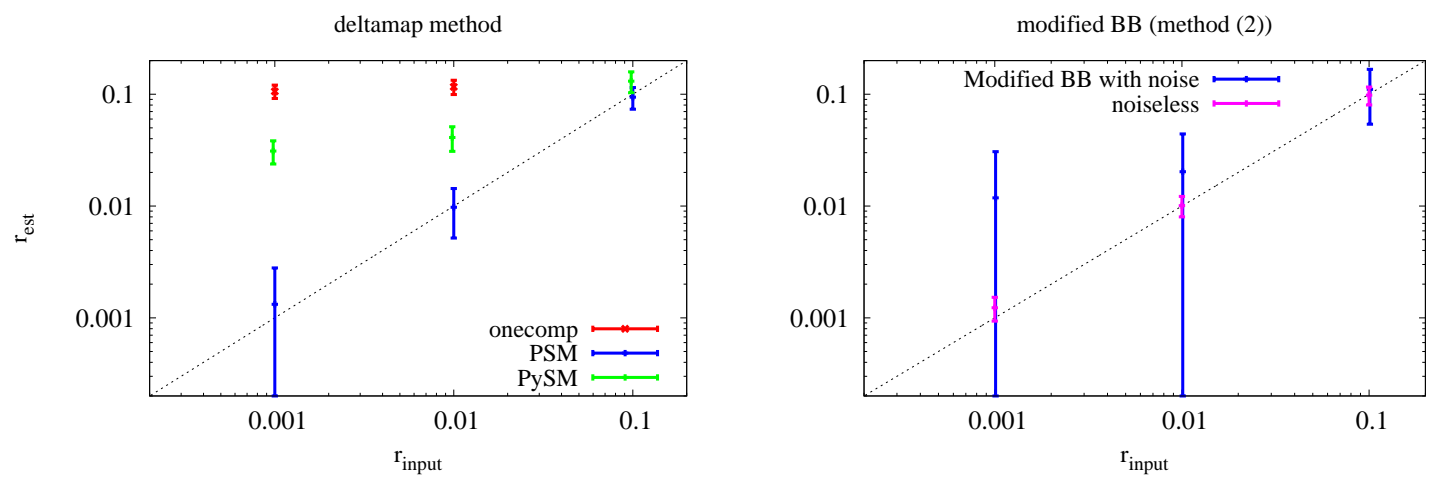

Figure 1: (Left) Estimated tensor-to-scalar ratio $r$ against the input, using the method (1), for various foreground models. PySM is another foreground model which we do not discuss in this poster. (Right) Estimated tensor-to-scalar ratio $r$ for the onecomp foreground model, using the method (2). In this case, the significant bias found in the left panel is reduced, but the boosted noise hinder the detection of the cosmological signal (blue). If we would switch off the noise, we recover the input signal using the method (2) (magenta).

\section{5. conclusion}

We have developed new template cleaning methods for the foreground removal problem in future CMB B-mode experiments. We have found that method (1) can remove the significant bias that was found in KK11 due to the spatial variation of spectral indices of the foreground emissions, and $r \approx 0.001$ is within reach, if the emissions are simple power-laws given by the Planck Sky Model. For the more complicated foreground model (onecomp), method (1) brings significant bias in estimating $r$ as large as $\delta r \approx 0.1$. Method (2) can remove the bias, but in this case instrumental noise dominates in the estimation of $r$ at the level of $\delta r \approx 0.01$.

\section{References}

[1] K. Ichiki, Progress of Theoretical and Experimental Physics 2014(6), 06B109, 06B109 (Jun. 2014).

[2] N. Katayama and E. Komatsu, ApJ 737, 78, 78 (Aug. 2011), 1101.5210. 